\title{
Long-term clinical outcomes after bilateral laminotomy or total laminectomy for lumbar spinal stenosis: a single-institution experience
}

\author{
Andrea Pietrantonio, MD, 1,3 Sokol Trungu, MD, ${ }^{1,2}$ Isabella Famà, MD, ${ }^{1}$ Stefano Forcato, MD,1,2 \\ Massimo Miscusi, MD, PhD, ${ }^{1}$ and Antonino Raco, MD, PhD1 \\ 1Department of Neuroscience, Mental Health, and Sense Organs, Faculty of Medicine and Psychology, "Sapienza" University of \\ Rome, Sant'Andrea Hospital, Rome; ${ }^{2}$ Neurosurgery Unit, Cardinale G. Panico Hospital, Tricase; and ${ }^{3}$ Neurosurgery Unit, Santa \\ Maria Goretti Hospital, Latina, Italy
}

\begin{abstract}
OBJECTIVE Lumbar spinal stenosis (LSS) is the most common spinal disease in the geriatric population, and is characterized by a compression of the lumbosacral neural roots from a narrowing of the lumbar spinal canal. LSS can result in symptomatic compression of the neural elements, requiring surgical treatment if conservative management fails. Different surgical techniques with or without fusion are currently treatment options. The purpose of this study was to provide a description of the long-term clinical outcomes of patients who underwent bilateral laminotomy compared with total laminectomy for LSS.
\end{abstract}

METHODS The authors retrospectively reviewed all the patients treated surgically by the senior author for LSS with total laminectomy and bilateral laminotomy with a minimum of 10 years of follow-up. Patients were divided into 2 treatment groups (total laminectomy, group 1; and bilateral laminotomy, group 2) according to the type of surgical decompression. Clinical outcomes measures included the visual analog scale (VAS), the 36-Item Short-Form Health Survey (SF-36) scores, and the Oswestry Disability Index (ODI). In addition, surgical parameters, reoperation rate, and complications were evaluated in both groups.

RESULTS Two hundred fourteen patients met the inclusion and exclusion criteria (105 and 109 patients in groups 1 and 2 , respectively). The mean age at surgery was 69.5 years (range $58-77$ years). Comparing pre- and postoperative values, both groups showed improvement in ODI and SF-36 scores; at final follow-up, a slightly better improvement was noted in the laminotomy group (mean ODI value 22.8, mean SF-36 value 70.2), considering the worse preoperative scores in this group (mean ODI value 70, mean SF-36 value 38.4) with respect to the laminectomy group (mean ODI 68.7 vs mean SF-36 value 36.3), but there were no statistically significant differences between the 2 groups. Significantly, in group 2 there was a lower incidence of reoperations $(15.2 \%$ vs $3.7 \%, p=0.0075)$.

CONCLUSIONS Bilateral laminotomy allows adequate and safe decompression of the spinal canal in patients with LSS; this technique ensures a significant improvement in patients' symptoms, disability, and quality of life. Clinical outcomes are similar in both groups, but a lower incidence of complications and iatrogenic instability has been shown in the long term in the bilateral laminotomy group.

https://thejns.org/doi/abs/10.3171/2019.2.FOCUS18651

KEYWORDS lumbar spinal stenosis; laminectomy; bilateral laminotomy

L UMBAR spinal stenosis (LSS) is defined as a narrowing of the lumbar spinal canal, and is one of the most common spine diseases in the geriatric population. LSS can result in symptomatic compression of the neural elements, requiring surgical treatment if conservative management fails.
Total laminectomy (initially known as a Christmas Tree laminectomy and now outdated) is the classic operative technique and consists of the unroofing of the spinal canal by resecting the spinous process, the laminae, the articular processes, and the pars interarticularis bilaterally; less invasive surgical options have been developed during

ABBREVIATIONS ASA = American Society of Anesthesiologists; DLSS = degenerative lumbar spinal stenosis; LOS = length of hospital stay; LSS = lumbar spinal stenosis; $\mathrm{ODI}=$ Oswestry Disability Index; SF-36 = 36-Item Short-Form Health Survey; VAS = visual analog scale.

SUBMITTED December 31, 2018. ACCEPTED February 26, 2019.

INCLUDE WHEN CITING DOI: 10.3171/2019.2.FOCUS18651. 
the past years, including bilateral laminotomy and unilateral laminotomy for bilateral decompression..$^{12,16,17,21} \mathrm{In}$ elderly patients with comorbidities, indirect decompression has been shown to be another treatment option. ${ }^{13}$ The main assumption that justifies the development of these techniques is the possibility to obtain an adequate decompression, minimizing the tissue trauma and preserving the spinal architecture. As a consequence, a better clinical outcome with minor long-term complications can be expected.

In the current study, we report the long-term clinical outcomes of patients who underwent bilateral laminotomy for degenerative LSS (DLSS); a comparison between this group and a group of patients treated with total laminectomy at our institution has been done and the results have been discussed and analyzed in detail.

\section{Methods \\ Patient Population}

We retrospectively reviewed all the patients treated surgically by the senior author (A.R.) for DLSS with total laminectomy and bilateral laminotomy; only the patients followed up for a minimum of 10 years and with complete clinical and radiological documentation were selected and are the subject of this study. Considering the progressive transition from the classic open procedures to minimally invasive approaches, we selected 2 time intervals, the first one (2000-2003) in which the classic laminectomies were routinely used and the second one (2005-2008), in which bilateral laminotomies were considered the procedure of choice by our group for the treatment of DLSS. We analyzed only the surgeries performed by the senior author, and we do not restrict our analysis to a single period in which both the procedures were performed, thus preventing the selection bias of considering one procedure more adequate than the other one during the surgical decisionmaking process. In fact, from 2005 we treated our patients almost exclusively with bilateral laminotomies, even when a severe compression was detected, whereas the use of lumbar laminectomies was restricted mainly to neoplastic and traumatic compressions.

Patients reviewed in the present study showed similar demographic data and comparable comorbidities and were divided into 2 treatment groups (total laminectomy, group 1 ; and bilateral laminotomy, group 2) according to the surgical decompression technique.

The diagnosis of LSS was made based on lumbar MRI or CT scanning (if MRI could not be done), if a single or multilevel compression was detected; flexion-extension radiographs were always performed to rule out any spine instability. From a clinical point of view, leg pain, leg numbness, and neurogenic claudication were considered more indicative than back pain, as well as improvement of symptoms during flexion of the trunk. Symptoms were considered refractory to nonsurgical treatment if conservative measures, particularly nonsteroidal antiinflammatory drugs and physical therapies, had been administered for at least 3 months without improvement.

For the purposes of this study, we excluded patients with previous spine surgery or fractures, spine instability, lumbar degenerative spondylolisthesis, degenerative scoliosis, developmental spinal deformities, neoplastic compression, and severe osteoporosis.

Preoperative clinical evaluation data were obtained from medical charts. Postoperative (3 weeks) and followup scores were obtained during outpatient visits or phone interviews 6,24, 60, and 120 months after surgery.

During the follow-up interviews, patients were asked about preoperative, immediate postoperative, and current status; improvement in symptoms; use of painkillers; patient satisfaction; and current therapy. Pain was measured according to a self-assessment 10-point visual analog scale (VAS).

The 36-Item Short-Form Health Survey (SF-36) and Oswestry Disability Index (ODI) were administered postoperatively (3 weeks) and at 6,24, 60, and 120 months after surgery. The SF-36 mental health and physical functioning summaries were analyzed in detail. The clinical outcomes were analyzed and statistical analyses performed with SPSS software v.15 (IBM Corp.). A p value < 0.05 was considered significant.

\section{Operative Technique}

All procedures were performed under general anesthesia; patients were placed prone on a Jackson spinal table with a Wilson frame support in a marked flexed position to reduce lumbar lordosis and increase the interlaminar space.

\section{Conventional Laminectomy (group 1)}

A 6-cm-long midline linear skin incision is made; the lumbodorsal fascia is incised vertically and the multifidus muscle is detached subperiosteally on each side and bilaterally retracted.

At the index level, the spinous process, the laminae, and the ligamentum flavum are resected. The medial portion of the facet joints (< one-third of the entire facet joint) is then removed bilaterally (facet-sparing laminectomy) to decompress the lateral recesses, using a high-speed drill and Kerrison rongeurs; foraminotomy is performed bilaterally. At the end of the decompression, suction drains are routinely placed; patients are generally allowed to walk with a corset brace within 2 days of surgery, and the use of a corset brace is recommended for 4-6 weeks.

\section{Bilateral Laminotomy (group 2)}

A 4-cm-long midline linear skin incision is made. The multifidus muscle from each side is detached from its attachments, the interlaminar space is exposed, and the retractors are applied.

With the aid of the surgical microscope, the upper and lower laminae at the index level are bilaterally thinned: the basal part of the spinous process, the caudal half of the cranial lamina, and a small cranial portion of the caudal lamina are then removed bilaterally by using a small Kerrison rongeur, starting from the upper lamina at the midline and then proceeding laterally. Care is taken not to detach the spinous process completely and to preserve the hypertrophied ligamentum flavum as long as possible for the protection of the dural sac and nerve roots during the 
osseous decompression. Following adequate bony resection, the ligamentum flavum is removed with a curette; partial resection of the facet joints and foraminotomy are usually done bilaterally to increase the neural decompression.

Generally, the intervertebral disc is not removed because in DLSS the compression acts mainly posteriorly and the preservation of the anterior stabilizing structures of the spine (intervertebral disc, posterior longitudinal ligament) is advisable. Suction drains are not routinely placed. Ambulation is encouraged on the day of surgery, and the use of a corset brace is not recommended.

\section{Results \\ Demographic Data}

Between January 2000 and January 2003, 172 patients underwent lumbar laminectomies for DLSS: among these, 105 patients (61\%) met the inclusion criteria and are the subject of this review (group 1). Similarly, between January 2005 and January 2008, 109 of the 168 patients $(64.9 \%)$ who underwent bilateral laminotomies met the inclusion criteria and were included in this review (group 2). The mean age of the entire cohort was 69.5 years (range 58-77 years); there were 117 women (54.7\%) and 97 men $(45.3 \%)(\mathrm{M} / \mathrm{F}$ ratio $1.2: 1)$

The duration of symptoms ranged from 6 months to 3 years prior to surgical intervention. The most common clinical symptom was neurogenic claudication, reported by the entire group (214 patients, 100\%), followed by low-back pain (70.6\%), radiculopathy (58.9\%), lower-limb weakness (55.6\%), and sphincteric dysfunction (5.1\%).

Of the 274 levels treated, the most commonly affected spinal level was L4-5 (142 patients, 51.8\%) followed by L3-4 (58 patients, 21.2\%), L5-S1 (15\%), L2-3 (8.4\%), and L1-2 (3.6\%). A double-level decompression was performed in 32 patients in group $1(30.5 \%)$ and in 30 patients $(27.5 \%)$ in group 2.

In the laminectomy group, the average surgical time and intraoperative blood loss per level were 52 minutes and $95 \mathrm{ml}$, respectively; the average postoperative blood loss from suction drains was $110 \mathrm{ml}$. In the laminotomy group, the average surgical time per level was a little longer (68 minutes), whereas the intraoperative blood loss was consistently lower $(50 \mathrm{ml})$. The average postoperative length of hospital stay (LOS) was shorter for the laminotomy group.

The comorbidity rate was high and mainly related to the mean age of our patients: 189 patients $(88.3 \%)$ were affected by several medical diseases, including cardiovascular diseases $(70.6 \%)$, respiratory diseases $(55.6 \%)$, diabetes mellitus (54.2\%), and obesity (38.8\%). The American Society of Anesthesiologists (ASA) score was as follows: none had ASA I, 25 (11.7\%) had ASA II, 143 (66.8\%) had ASA III, and 46 (21.5\%) had ASA IV.

All patients' medical records, including clinical and radiological data, are summarized in Table 1 .

\section{Clinical Outcomes}

The mean preoperative VAS scores were 7.9 and 8.3 in the bilateral laminotomy and open laminectomy groups, respectively. At final follow-up, the improvement was greater in the laminotomy group (mean value 3.6) than in the laminectomy group (mean value 4.4), and a similar difference was noted at each follow-up visit.

Comparing pre- and postoperative values, both groups showed improvement in ODI and SF-36 scores. At final follow-up, a greater improvement was noted in the laminotomy group (mean ODI value 22.8, mean SF-36 value 70.2 ), considering the worse preoperative scores (mean ODI value 70, mean SF-36 value 38.4) with respect to the laminectomy group (mean ODI value 68.7, mean SF-36 value 36.3). However, there was no statistically significant difference in clinical outcomes (VAS, ODI, SF-36) between the 2 groups.

In both groups, none of the patients required postoperative rehabilitation. All patients' clinical outcomes are summarized in Table 2.

\section{Reoperation Rate and Complications}

In 9 cases (8.6\%) in group 1 and in 5 cases (4.6\%) in group 2, an incidental durotomy was reported. In the laminectomy group, the dural tear could be identified in all patients and directly repaired at surgery, whereas in 3 patients in the laminotomy group, the point of leakage could not be clearly seen and a dural sealant instead of direct suture had to be used. In these 14 patients, 3 days of postoperative bed rest was advised and no CSF fistula developed after surgery.

We observed 5 superficial wound infections (2.3\% of the entire cohort), which resolved with antibiotic therapy and daily medications; 4 (3.8\%) in group 1 and 1 (0.9\%) in group 2.

During the follow-up period, 4 patients (3.7\%) in group 2 underwent reoperation because of inadequate decompression; after a transient and partial relief of symptoms, a second operation had to be performed because of clinical deterioration and MRI evidence of lumbar stenosis at the treated level. All these patients underwent reoperation within 18 months of the first surgery.

In group 1, 16 patients $(15.2 \%)$ underwent subsequent lumbar fusion surgery within 38 months: in 15 patients (93.7\%), lumbar fusion was required to treat a postlaminectomy instability, whereas in the remaining patient a second decompression and fusion was performed to treat a residual stenosis $(p=0.0075)$.

All patients' reoperation rates and complications are summarized in Table 3.

\section{Discussion}

The incidence of LSS ranges between $3.9 \%$ and $11 \%$ of the general population, and its prevalence is steadily growing in our aging society. LSS may be a developmental or an acquired disease, the last one being far more common, and generally has a slowly progressive course. LSS is caused by a compression of the neural elements from different structures of the spinal canal: ligamentum flavum hypertrophy and disc bulging may lead to central stenosis, whereas facet hypertrophy and settling are associated with recess or foraminal compression. These degenerative changes are mostly age related and may pro- 
TABLE 1. Details of the final cohort of 214 patients with LSS

\begin{tabular}{|c|c|c|c|}
\hline Characteristic & Group 1, Laminectomy & Group 2, Laminotomy & Overall \\
\hline Total no. of patients & $105(49 \%)$ & $109(51 \%)$ & 214 \\
\hline Mean age in yrs (range) & 68.5 & 70.1 & $69.5(58-77)$ \\
\hline \multicolumn{4}{|l|}{ Sex } \\
\hline Female & $58(55.2 \%)$ & $59(54.1 \%)$ & $117(54.7 \%)$ \\
\hline Male & $47(44.8 \%)$ & $50(45.9 \%)$ & $97(45.3 \%)$ \\
\hline \multicolumn{4}{|l|}{ ASA classification } \\
\hline I & 0 & 0 & 0 \\
\hline II & $10(9.5 \%)$ & $15(13.8 \%)$ & $25(11.7 \%)$ \\
\hline III & $68(64.8 \%)$ & $75(68.8 \%)$ & $143(66.8 \%)$ \\
\hline IV & $27(25.7 \%)$ & $19(17.4 \%)$ & $46(21.5 \%)$ \\
\hline \multicolumn{4}{|l|}{ Level of stenosis* } \\
\hline L1-2 & $5(3.7 \%)$ & $5(3.6 \%)$ & $10(3.6 \%)$ \\
\hline L2-3 & $10(7.4 \%)$ & $13(9.4 \%)$ & $23(8.4 \%)$ \\
\hline L3-4 & $30(22.1 \%)$ & $28(20.3 \%)$ & $58(21.2 \%)$ \\
\hline L4-5 & $70(51.4 \%)$ & $72(52.2 \%)$ & $142(51.8 \%)$ \\
\hline L5-S1 & $21(15.4 \%)$ & $20(14.5 \%)$ & $41(15 \%)$ \\
\hline \multicolumn{4}{|l|}{ Levels treated } \\
\hline 1 level & $73(69.5 \%)$ & $79(72.5 \%)$ & $152(71 \%)$ \\
\hline 2 levels & $32(30.5 \%)$ & $30(27.5 \%)$ & $62(29 \%)$ \\
\hline Mean length of op (range) & 52 mins & 68 mins & 59 mins (45-110 mins) \\
\hline Mean LOS (range) & 3 days & 2 days & 2.5 days ( $1-5$ days) \\
\hline Mean time to postop mobilization (range) & 1 day & 1 day & 1 day (1-4 days) \\
\hline Intraop blood loss (range) & $95 \mathrm{ml}$ & $50 \mathrm{ml}$ & $72 \mathrm{ml}(40-180 \mathrm{ml})$ \\
\hline \multicolumn{4}{|l|}{ Symptoms } \\
\hline Low-back pain & $74(70.5 \%)$ & $77(70.6 \%)$ & $151(70.6 \%)$ \\
\hline Neurogenic claudication & $105(100 \%)$ & $109(100 \%)$ & $214(100 \%)$ \\
\hline Radiculopathy & $60(57.1 \%)$ & $66(60.6 \%)$ & $126(58.9 \%)$ \\
\hline Lower-limb weakness & $57(54.3 \%)$ & $62(56.9 \%)$ & $119(55.6 \%)$ \\
\hline Sphincteric dysfunction & $5(4.8 \%)$ & $6(5.5 \%)$ & $11(5.1 \%)$ \\
\hline \multicolumn{4}{|l|}{ Comorbidity } \\
\hline Cardiovascular diseases & $72(68.6 \%)$ & $79(72.5 \%)$ & $151(70.6 \%)$ \\
\hline Diabetes mellitus & $55(52.4 \%)$ & $61(56 \%)$ & $116(54.2 \%)$ \\
\hline Obesity & $39(37.1 \%)$ & $44(40.4 \%)$ & $83(38.8 \%)$ \\
\hline Respiratory diseases & $42(40 \%)$ & $52(47.7 \%)$ & $119(55.6 \%)$ \\
\hline
\end{tabular}

duce a vascular and/or mechanical compression on the neural roots. ${ }^{2,12,24}$

Classically, the surgical treatment of lumbar stenosis has been represented by a wide posterior decompression of the spinal canal: until the last few years, the standard procedure has been a wide laminectomy with a partial or complete removal of the articular processes. ${ }^{6,8,10}$ This procedure destroys the entire posterior bony arch and posterior ligamentous complex, and it detaches the paraspinal muscles bilaterally, thus damaging significantly the posterior stabilizing structures of the spine. Not surprisingly, the short-term neurological outcome after a laminectomy is generally good, because of the wide decompression achievable, but concerns regarding postoperative insta- bility and subsequent low-back pain in the medium and long term have been raised. Different studies have reported a higher iatrogenic instability following laminectomy compared to minimally invasive decompression., ${ }^{1,20} \mathrm{On}$ the basis of these and other experiences, a new trend in spine surgery, consisting of minimizing the normal tissue trauma while ensuring the same clinical, radiological, and neurological outcome, has progressively emerged..$^{13,16}$

Unilateral and bilateral laminotomy for decompression of the lumbar spine was introduced in 1981 by Getty et al. as less invasive surgical options. ${ }^{5}$ Laminotomy involves the partial removal of bone from the inferior aspect of the superior lamina, the superior portion of the inferior lamina, and the medial aspect of the facet joint, and it also 
TABLE 2. Clinical outcomes in both groups of patients with LSS

\begin{tabular}{lccc}
\hline Scoring System & $\begin{array}{c}\text { Group 1, } \\
\text { Laminectomy }\end{array}$ & $\begin{array}{c}\text { Group 2, } \\
\text { Laminotomy }\end{array}$ & $\begin{array}{c}\text { (group 1 } \\
\text { vs 2) }\end{array}$ \\
\hline VAS & & & 0.35 \\
\hline Preop (mean score) & 8.3 & 7.9 & \\
\hline Postop (3 wks) & 4.2 & 3.1 & \\
\hline Follow-up at 6 mos & 3.6 & 2.7 & \\
\hline Follow-up at 2 yrs & 3.5 & 2.8 & \\
\hline Follow-up at 5 yrs & 4.1 & 3.4 & \\
\hline Follow-up at 10 yrs & 4.4 & 3.6 & \\
\hline p value (pre vs post) & 0.002 & 0.001 & \\
\hline ODI & & & \\
\hline Preop (mean score) & 68.7 & 70 & \\
\hline Postop (3 wks) & 37.5 & 32.6 & \\
\hline Follow-up at 6 mos & 16.8 & 16.2 & \\
\hline Follow-up at 2 yrs & 17.5 & 18.3 & \\
\hline Follow-up at 5 yrs & 23.5 & 20.1 & \\
\hline Follow-up at 10 yrs & 24.6 & 22.8 & \\
\hline p value (pre vs post) & 0.003 & 0.002 & \\
\hline SF-36 (Physical and Mental) & & & \\
\hline Preop (mean score) & 36.3 & 38.4 & \\
\hline Postop (3 wks) & 64.8 & 63.9 & \\
\hline Follow-up at 6 mos & 74.2 & 75.3 & \\
\hline Follow-up at 2 yrs & 77.1 & 78.2 & \\
\hline Follow-up at 5 yrs & 72.2 & 74.3 & \\
\hline Follow-up at 10 yrs & 69.1 & 70.2 & \\
\hline p value (pre vs post) & 0.001 & 0.003 & \\
\hline & & & \\
\hline
\end{tabular}

entails the excision of the ligamentum flavum; the spinous process and supra- and interspinous ligaments are preserved. ${ }^{18,25,26}$ The unilateral approach for bilateral decompression preserves the contralateral structures (lamina and facet joint) and ensures enough contralateral decompression by drilling the base of the spinous process and by tilting the microscope and the operating table.

Different studies showed no difference in clinical outcome between bilateral laminotomy and laminectomy. ${ }^{9,19,22}$ In their multicenter observational study, Nerland et al. compared minimally invasive microdecompression and open laminectomy for the treatment of central lumbar stenosis and reported similar outcome scores at 1-year follow-up and a shorter hospital stay for the minimally

TABLE 3. Complications and reoperation rate in both groups of patients with LSS

\begin{tabular}{lccc}
\hline \multicolumn{1}{c}{ Variable } & $\begin{array}{c}\text { Group 1, } \\
\text { Laminectomy }\end{array}$ & $\begin{array}{c}\text { Group 2, } \\
\text { Laminotomy }\end{array}$ & $\begin{array}{c}\text { p Value } \\
\text { (group 1 vs 2) }\end{array}$ \\
\hline Complications & & & \\
\hline Wound infections & $4(3.8 \%)$ & $1(0.9 \%)$ & 0.89 \\
\hline Durotomy & $9(8.6 \%)$ & $5(4.6 \%)$ & 0.81 \\
\hline Reop rate & $16(15.2 \%)$ & $4(3.7 \%)$ & 0.0075 \\
\hline
\end{tabular}

invasive group. Considering the similar effectiveness of these techniques, the authors concluded that minimally invasive decompression should be favored when there are concerns about later instability. ${ }^{15}$ Several studies showed that minimally invasive techniques ensure at least the same results as the standard open technique; moreover, results are better in terms of clinical improvement, LOS, blood loss, time to mobilization, use of medications, and patients' satisfaction in the short and medium term., $, 14,17$

Defining instability as sagittal plane translation $\geq 5 \mathrm{~mm}$ on dynamic radiographs, Thomé et al. reported a frequency of iatrogenic instability following laminectomy and unilateral laminotomy for bilateral decompression of $8.8 \%$ and $5.1 \%$, respectively. Conversely, none of their patients treated with bilateral laminotomy showed radiographic and clinical evidence of postoperative instability. ${ }^{23}$ On the other hand, in the retrospective study of Costa et al., 3 of 374 patients $(0.8 \%)$ treated via unilateral laminotomy for bilateral decompression experienced postoperative back pain suggestive of iatrogenic instability. ${ }^{3}$ In 2010, Lee et al. used cadaveric lumbar spine models to study the range of motion after bilateral laminotomy and facet-sparing laminectomy; the analysis per level demonstrated a roughly 2 -fold increase in motion after laminectomy compared with bilateral laminotomies (14.3\% in bilateral laminotomies vs $32.0 \%$ in full laminectomies). Moreover, stiffness was decreased by an average of $11.8 \%$ after 3-level laminotomies and by $27.2 \%$ after 3 -level laminectomies. ${ }^{11}$

As a result of all these experiences and because of the concerns for late instability and the comparable clinical outcome, the classic wide laminectomy with facetectomy has been more frequently replaced with minimally invasive techniques. This is also our experience-in recent years fewer cases of laminectomies have been performed by our group for degenerative diseases, whereas the number of patients treated with bilateral laminotomies has continuously increased. Our results confirm the evidence emerging in the literature in the last few years and reported in this paper: in the laminotomy group, LOS and intraoperative blood loss were less.

Regarding complications, we observed a little increase in the frequency of dural tears in the laminotomy group. We found it more difficult to repair the leakage with sutures, because the smaller operative corridor makes both the suturing and the identification of the tear more challenging. However, the use of sealant and a more prolonged postoperative bed rest have proved to be effective in preventing CSF leakage. A higher incidence of incomplete decompression requiring reintervention was observed in the laminotomy group (3.7\% vs $1 \%$ ); we explain this as an error in surgical indication, because all these patients showed a severe foraminal stenosis that would have been better treated with a wide artrectomy and fusion from the beginning. No patient with postoperative instability was seen in the laminotomy group, as compared to the laminectomy group, in which 15 patients (14.3\%) underwent fixation for iatrogenic listhesis and back pain.

Regarding clinical outcomes, the success rate of the 2 techniques was similar, although a slightly better outcome and higher patient satisfaction after bilateral laminotomy can be seen; in the medium to long term, bilateral laminot- 
omy proved to be equally effective in improving pain and function if compared to standard decompression. However, the main advantages of this less invasive technique consist of the reduction of postoperative instability, ensuring at the same time an adequate degree of decompression.

\section{Conclusions}

Bilateral laminotomy allows adequate and safe decompression of the spinal canal in patients with lumbar stenosis; this technique ensures a significant improvement in patients' symptoms, disability, and quality of life. Neurological outcome after bilateral laminotomy and laminectomy is similar, but a lower incidence of complications and iatrogenic instability has been shown in the medium and long term for patients who undergo bilateral laminotomy.

\section{References}

1. Abumi K, Panjabi MM, Kramer KM, Duranceau J, Oxland T, Crisco JJ: Biomechanical evaluation of lumbar spinal stability after graded facetectomies. Spine (Phila Pa 1976) 15:1142-1147, 1990

2. Choi WS, Oh CH, Ji GY, Shin SC, Lee JB, Park DH, et al: Spinal canal morphology and clinical outcomes of microsurgical bilateral decompression via a unilateral approach for lumbar spinal canal stenosis. Eur Spine J 23:991-998, 2014

3. Costa F, Sassi M, Cardia A, Ortolina A, De Santis A, Luccarell G, et al: Degenerative lumbar spinal stenosis: analysis of results in a series of 374 patients treated with unilateral laminotomy for bilateral microdecompression. J Neurosurg Spine 7:579-586, 2007

4. Fu YS, Zeng BF, Xu JG: Long-term outcomes of two different decompressive techniques for lumbar spinal stenosis. Spine (Phila Pa 1976) 33:514-518, 2008

5. Getty CJ, Johnson JR, Kirwan EO, Sullivan MF: Partial undercutting facetectomy for bony entrapment of the lumbar nerve root. J Bone Joint Surg Br 63-B:330-335, 1981

6. Grabias S: Current concepts review. The treatment of spinal stenosis. J Bone Joint Surg Am 62:308-313, 1980

7. Guha D, Heary RF, Shamji MF: Iatrogenic spondylolisthesis following laminectomy for degenerative lumbar stenosis: systematic review and current concepts. Neurosurg Focus 39(4): E9, 2015

8. Johnsson KE, Redlund-Johnell I, Udén A, Willner S: Preoperative and postoperative instability in lumbar spinal stenosis. Spine (Phila Pa 1976) 14:591-593, 1989

9. Kalbarczyk A, Lukes A, Seiler RW: Surgical treatment of lumbar spinal stenosis in the elderly. Acta Neurochir (Wien) 140:637-641, 1998

10. Lane WA: Case of spondylolisthesis associated with progressive paraplegia: laminectomy. Lancet 1:991, 1893

11. Lee MJ, Bransford RJ, Bellabarba C, Chapman JR, Cohen AM, Harrington RM, et al: The effect of bilateral laminotomy versus laminectomy on the motion and stiffness of the human lumbar spine: a biomechanical comparison. Spine (Phila Pa 1976) 35:1789-1793, 2010

12. Mayer HM: Minimally Invasive Spine Surgery. A Surgical Manual, ed 2. Berlin: Springer, 2005

13. Miscusi M, Trungu S, Forcato S, Ramieri A, Polli FM, Raco A: Long-term clinical outcomes and quality of life in elderly patients treated with interspinous devices for lumbar spinal stenosis. J Neurol Surg A Cent Eur Neurosurg 79:139-144, 2018

14. Mobbs RJ, Li J, Sivabalan P, Raley D, Rao PJ: Outcomes after decompressive laminectomy for lumbar spinal stenosis: comparison between minimally invasive unilateral laminectomy for bilateral decompression and open laminectomy: clinical article. J Neurosurg Spine 21:179-186, 2014
15. Nerland US, Jakola AS, Solheim O, Weber C, Rao V, Lønne $\mathrm{G}$, et al: Minimally invasive decompression versus open laminectomy for central stenosis of the lumbar spine: pragmatic comparative effectiveness study. BMJ 350:h1603, 2015

16. Oppenheimer JH, DeCastro I, McDonnell DE: Minimally invasive spine technology and minimally invasive spine surgery: a historical review. Neurosurg Focus 27(3):E9, 2009

17. Phan K, Mobbs RJ: Minimally invasive versus open laminectomy for lumbar stenosis: a systematic review and metaanalysis. Spine (Phila Pa 1976) 41:E91-E100, 2016

18. Poletti CE: Central lumbar stenosis caused by ligamentum flavum: unilateral laminotomy for bilateral ligamentectomy: preliminary report of two cases. Neurosurgery 37:343-347, 1995

19. Postacchini F, Cinotti G, Perugia D, Gumina S: The surgical treatment of central lumbar stenosis. Multiple laminotomy compared with total laminectomy. J Bone Joint Surg Br 75:386-392, 1993

20. Ramhmdani S, Xia Y, Xu R, Kosztowski T, Sciubba D, Witham T, et al: Iatrogenic spondylolisthesis following open lumbar laminectomy: case series and review of the literature. World Neurosurg 113:e383-e390, 2018

21. Schöller K, Steingrüber T, Stein M, Vogt N, Müller T, PonsKühnemann J, et al: Microsurgical unilateral laminotomy for decompression of lumbar spinal stenosis: long-term results and predictive factors. Acta Neurochir (Wien) 158:11031113,2016

22. Thomas NW, Rea GL, Pikul BK, Mervis LJ, Irsik R, McGregor JM: Quantitative outcome and radiographic comparisons between laminectomy and laminotomy in the treatment of acquired lumbar stenosis. Neurosurgery 41:567-575, 1997

23. Thomé C, Zevgaridis D, Leheta O, Bäzner H, Pöckler-Schöniger $\mathrm{C}$, Wöhrle J, et al: Outcome after less-invasive decompression of lumbar spinal stenosis: a randomized comparison of unilateral laminotomy, bilateral laminotomy, and laminectomy. J Neurosurg Spine 3:129-141, 2005

24. Ullrich CG, Binet EF, Sanecki MG, Kieffer SA: Quantitative assessment of the lumbar spinal canal by computed tomography. Radiology 134:137-143, 1980

25. Weiner BK, Walker M, Brower RS, McCulloch JA: Microdecompression for lumbar spinal canal stenosis. Spine (Phila Pa 1976) 24:2268-2272, 1999

26. Young S, Veerapen R, O'Laoire SA: Relief of lumbar canal stenosis using multilevel subarticular fenestrations as an alternative to wide laminectomy: preliminary report. Neurosurgery 23:628-633, 1988

\section{Disclosures}

The authors report no conflict of interest concerning the materials or methods used in this study or the findings specified in this paper.

\section{Author Contributions}

Conception and design: Trungu, Pietrantonio, Famà, Miscusi, Raco. Acquisition of data: Trungu, Pietrantonio, Famà. Analysis and interpretation of data: Trungu, Pietrantonio, Famà. Drafting the article: Trungu, Pietrantonio. Critically revising the article: Forcato, Miscusi, Raco. Reviewed submitted version of manuscript: Pietrantonio, Forcato, Miscusi, Raco. Approved the final version of the manuscript on behalf of all authors: Trungu. Statistical analysis: Pietrantonio, Forcato. Study supervision: Raco.

\section{Correspondence}

Sokol Trungu: "Sapienza" University of Rome, Sant'Andrea Hospital, Rome, Italy. s_trungu@hotmail.com. 